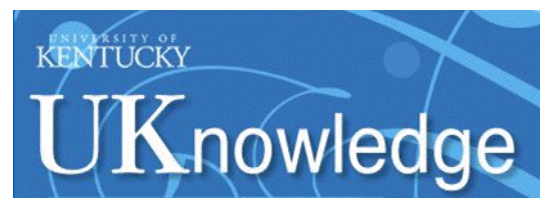

University of Kentucky

UKnowledge

$9-30-2020$

\title{
Unveiling the Molecular Mechanism of SARS-CoV-2 Main Protease Inhibition from 137 Crystal Structures Using Algebraic Topology and Deep Learning
}

Duc Duy Nguyen

University of Kentucky, ducnguyen@uky.edu

Kaifu Gao

Michigan State University

Jiahui Chen

Michigan State University

Rui Wang

Michigan State University

Guo-Wei Wei

Michigan State University

Follow this and additional works at: https://uknowledge.uky.edu/math_facpub

Part of the Biochemistry, Biophysics, and Structural Biology Commons, and the Mathematics

Commons

Right click to open a feedback form in a new tab to let us know how this document benefits you.

\section{Repository Citation}

Nguyen, Duc Duy; Gao, Kaifu; Chen, Jiahui; Wang, Rui; and Wei, Guo-Wei, "Unveiling the Molecular Mechanism of SARS-CoV-2 Main Protease Inhibition from 137 Crystal Structures Using Algebraic Topology and Deep Learning" (2020). Mathematics Faculty Publications. 45.

https://uknowledge.uky.edu/math_facpub/45

This Article is brought to you for free and open access by the Mathematics at UKnowledge. It has been accepted for inclusion in Mathematics Faculty Publications by an authorized administrator of UKnowledge. For more information, please contact UKnowledge@lsv.uky.edu. 
Unveiling the Molecular Mechanism of SARS-CoV-2 Main Protease Inhibition from 137 Crystal Structures Using Algebraic Topology and Deep Learning

Digital Object Identifier (DOI)

https://doi.org/10.1039/d0sc04641h

Notes/Citation Information

Published in Chemical Science, v. 11, issue 44.

(C) The Royal Society of Chemistry 2020

This article is licensed under a Creative Commons Attribution-NonCommercial 3.0 Unported Licence. 


\section{Chemical Science}

Check for updates

Cite this: Chem. Sci., 2020, 11, 12036

๑ All publication charges for this article have been paid for by the Royal Society of Chemistry

Received 21st August 2020

Accepted 30th September 2020

DOI: $10.1039 / \mathrm{dOsc04641h}$

rsc.li/chemical-science

\section{Unveiling the molecular mechanism of SARS-CoV-2 main protease inhibition from 137 crystal structures using algebraic topology and deep learning $\dagger$}

\author{
Duc Duy Nguyen, ${ }^{a}$ Kaifu Gao, ${ }^{b}$ Jiahui Chen, ${ }^{b}$ Rui Wang ${ }^{b}$ and Guo-Wei Wei (D)*bcd
}

Currently, there is neither effective antiviral drugs nor vaccine for coronavirus disease 2019 (COVID-19) caused by acute respiratory syndrome coronavirus 2 (SARS-CoV-2). Due to its high conservativeness and low similarity with human genes, SARS-CoV-2 main protease $\left(\mathrm{M}^{\mathrm{pro}}\right.$ ) is one of the most favorable drug targets. However, the current understanding of the molecular mechanism of $M^{\text {pro }}$ inhibition is limited by the lack of reliable binding affinity ranking and prediction of existing structures of $M^{\text {pro-inhibitor }}$ complexes. This work integrates mathematics (i.e., algebraic topology) and deep learning (MathDL) to

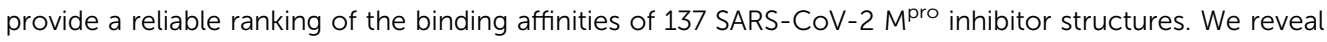
that Gly143 residue in $M^{\text {pro }}$ is the most attractive site to form hydrogen bonds, followed by Glu166, Cys145, and His163. We also identify 71 targeted covalent bonding inhibitors. MathDL was validated on the PDBbind v2016 core set benchmark and a carefully curated SARS-CoV-2 inhibitor dataset to ensure the reliability of the present binding affinity prediction. The present binding affinity ranking, interaction analysis, and fragment decomposition offer a foundation for future drug discovery efforts.

\section{Introduction}

Starting in late Dec, 2019, the COVID-19 pandemic caused by new severe acute respiratory syndrome coronavirus (SARS-CoV2) has infected more than 22 million individuals and has caused more than 777000 fatalities in all of the continents and over 213 countries and territories by August 19th, 2020. Under the current global health emergency, researchers around the world have engaged in the investigation of the different drug targets of SARS-CoV-2, such as the main protease $\left(\mathrm{M}^{\text {pro }}\right.$, also called $\left.3 \mathrm{CL}^{\text {pro }}\right)$, papain-Like protease $\left(\mathrm{PL}^{\text {pro }}\right)$, RNA-dependent RNA polymerase (RdRp), 5'-to-3' helicase protein (Nsp13) to seek potential cures for this serious pandemic. To date, although there are some vaccines undergoing the Phase III trials, ${ }^{1}$ their safety and efficacy are still unclear. ${ }^{2}$

The main protease, one of the best-characterized targets for coronaviruses, attracts lots of research attention because it is

${ }^{a}$ Department of Mathematics, University of Kentucky, KY 40506, USA

${ }^{b}$ Department of Mathematics, Michigan State University, MI 48824, USA. E-mail: weig@msu.edu

${ }^{c}$ Department of Biochemistry and Molecular Biology, Michigan State University, MI 48824, USA

${ }^{d}$ Department of Electrical and Computer Engineering, Michigan State University, MI 48824, USA

$\dagger$ Electronic supplementary information (ESI) available: SupportingTables.xls: spreadsheets contain information for all supporting tables from S1 to S8; FileS1.zip: 3D structures generated by our MathPose for 141 ligands in SARS-CoV 2D set; FigS1.pdf: deep learning architecture of MathDL model. See DOI: $10.1039 / \mathrm{d} 0 \mathrm{sc} 04641 \mathrm{~h}$ very conservative and distinguished from any human gene. A recent study shows that although the overall sequence identity between SARS-CoV and SARS-CoV-2 is just $80 \%$, the $\mathrm{M}^{\text {pro }}$ of SARS-CoV-2 shares $96.08 \%$ sequence identity to that of SARS$\mathrm{CoV}^{3}{ }^{3}$ Therefore, we hypothesize that a potent SARS $\mathrm{M}^{\text {pro }}$ inhibitor is also a potent SARA-CoV-2 $\mathrm{M}^{\text {pro }}$ inhibitor.

At this moment, more than 300 potential SARS-CoV $\mathrm{M}^{\text {pro }}$ inhibitors with its binding affinities are available in ChEMBL database ${ }^{4}$ which can be considered as the potential SARS-CoV-2 $\mathrm{M}^{\text {pro }}$ inhibitors. Recently, total 146 crystal structures of SARS$\mathrm{CoV}-2 \mathrm{M}^{\text {pro }}$ with its ligand complexes are released on the Protein Data Bank (PDB). ${ }^{5}$ Among them, 137 crystal structures have no available binding affinities reported for various reasons. However, the central dogma of drug design and discovery concerns the molecular mechanism and binding affinity of drug target interactions. Knowing the binding affinities and their ranking of 137 SARS-CoV-2 $\mathrm{M}^{\text {pro }}$ inhibitors is of great significance to the future design of anti SARS-CoV-2 drugs.

In this work, for the first time, we predict the binding

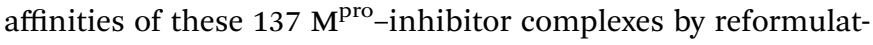
ing algebraic topology-based mathematics-deep learning (MathDL) models, which have been the top competitor in D3R Grand Challenges, a worldwide competition series in computer aided drug design in the past three years. ${ }^{6}$ We generate reliable poses for $141 \mathrm{M}^{\text {pro }}$ inhibitors with binding affinities but without complex structures. Together with 44 other complexes, we compose a set of $185 \mathrm{M}^{\text {pro }}$-inhibitor complexes, which is paired with 17382 protein-ligand complexes in PDBbind 2019 general set. These datasets are utilized to construct 11 MathDL models 
in single-task and multitask settings. ${ }^{6}$ One of these 11 MathDL models has been validated by using the PDBbind v2016 core set benchmark, achieving the top performance over all exiting scoring functions. The other ten MathDL models have crossvalidated on a set of $185 \mathrm{M}^{\text {pro }}$-nhibitor complexes, showing an averaged Pearson's correlation coefficient of 0.73 .

Notably, for covalent inhibitors, the scheme of covalent irreversible inhibition of SARS-CoV/SARS-CoV-2 $\mathbf{M}^{\text {pro }}$ is presented below:

$$
\mathrm{E}+\mathrm{I} \underset{k_{2}}{\stackrel{k_{1}}{\rightleftharpoons}} \mathrm{EI} \stackrel{k_{3}}{\longrightarrow} \mathrm{E}-\mathrm{I}
$$

The inhibitor first binds to the protease noncovalently, then a nucleophilic attacking by Cys145 leads to the formation of a stable covalent bond between the protease and the inhibitor. ${ }^{7,8}$ The interaction depends on both the equilibrium-binding constant $K_{\mathrm{i}}$ (designated as $k_{1} / k_{2}$ ) and the inactivation rate constant for covalent bond formation $k_{3}$. In this work, the binding affinity/ $\mathrm{IC}_{50}$ assesses the first step to form noncovalent binding.

In a nutshell, the present work provides reliable binding affinity predictions and ranking of 137 SARS-CoV-2 inhibitors that have crystal structures. It also offers data curation and validated models for exploring potential SARS-CoV-2 $\mathbf{M}^{\text {pro }}$ inhibitors. Furthermore, this work explores different possible binding regions on the SARS-CoV-2 main protease and decode the most favorable molecular fragments for the inhibitor design.

\section{Results and discussions}

\subsection{Results}

This section is devoted to the utilization of our MathDL models developed in Section 3.3 to predict the binding affinities and their ranking of SARS-CoV-2 inhibitors that do not have reported experimental affinities. To reduce the role of $3 \mathrm{D}$ pose prediction errors in our model, we use the SARS-CoV-2 inhibitors with X-ray structures available in the PDB for our study. We manually search these ligands on the PDB and arrive at a set consisting of 137 SARS-CoV-2 $\mathrm{M}^{\text {pro }}$ inhibitors having X-ray crystal structures but lacking of experimental binding affinities. We name this set SARS-CoV PDB-noBA (see Table 3). In this experiment, we develop a MathDL model optimized from PDBbind v2016 core set (see Section 3.3.1), five MathDL-ALL and five MathDL-MT models obtained from 5-fold study on the SARS-CoV BA set (see Section 3.3.2). The final predicted binding affinity is the consensus of these 11 models. The top ten inhibitors indicated by our models are shown in Table 1.

The most potent SARS-CoV-2 inhibitor found by our MathDL models is the inhibitor Nol in complex 7c8t. Nol was synthesized by Yang and his colleagues, ${ }^{9}$ Nol is found remarkable activities against SARS-CoV and $\mathrm{HCoV} .{ }^{9}$ Specifically, the dissociation constant $K_{\mathrm{i}}$ of Nol was found to be $0.053 \mu \mathrm{M}$ against SARS-CoV. ${ }^{9}$ Our MathDL reveals that Nol still inhibits SARSCoV-2 main protease with a potent affinity at $0.30 \mu \mathrm{M}$.
Table 1 Binding affinities of top 10 complexes in SARS-CoV PBDnoBA dataset predicted by our MathDL. "Pred. BA" indicates the predicted binding free energy in $\mathrm{kcal} \mathrm{mol}^{-1}$ and "Pred. $\mathrm{IC}_{50}$ " is the corresponding $I C_{50}$ in $\mu \mathrm{M}$ unit via the following conversion: Pred. $I C_{50}=$ $10^{\text {Pred. BA/1.3633 }} \times 10^{6}$

\begin{tabular}{|c|c|c|c|c|c|}
\hline PDBID & Pred. BA & $\begin{array}{l}\text { Pred. } \\
\text { IC }_{50}\end{array}$ & PDBID & Pred. BA & $\begin{array}{l}\text { Pred. } \\
\text { IC }_{50}\end{array}$ \\
\hline $7 c 8 t$ & -8.90 & 0.30 & $6 z 2 e$ & -8.43 & 0.66 \\
\hline 5rgl & -8.50 & 0.58 & $6 x b i$ & -8.34 & 0.76 \\
\hline $6 \times \mathrm{xm}$ & -8.50 & 0.58 & $6 x m k$ & -8.33 & 0.78 \\
\hline 7bqy & -8.49 & 0.59 & $5 \mathrm{rh} 7$ & -8.32 & 0.79 \\
\hline $5 \mathrm{rfr}$ & -8.45 & 0.63 & $6 x b h$ & -8.27 & 0.86 \\
\hline
\end{tabular}

Another important top potent SARS-CoV-2 inhibitor found by our models is the Michael acceptor inhibitor N3 in complex 7bqy. Designed by Yang and his colleagues, ${ }^{8} \mathrm{~N} 3$ was found to have viral activities against different coronavirus $\mathbf{M}^{\text {pro }}$ such as SARS-CoV and MERS-CoV. ${ }^{\mathbf{8 1 0}}$ Specifically, the dissociation constant $K_{\mathrm{i}}$ of $\mathrm{N} 3$ was found to be $9.0 \mu \mathrm{M}$ against SARS-CoV. ${ }^{8}$ Our MathDL reveals that N3 still inhibits SARS-CoV-2 main protease with an even better affinity at $0.59 \mu \mathrm{M}$. This finding is consistent with the literature work ${ }^{\mathbf{1 1}}$ showing that N3 is a potent inhibitor of COVID-19 virus $\mathrm{M}^{\text {pro }}$.

The inhibitor Qys in the complex 6xmk is also noticeable. Our predicted $\mathrm{IC}_{50}$ is $0.78 \mu \mathrm{M}$. Soon after we made the prediction, on August 12th, 2020, Rathnayake et al. ${ }^{12}$ released another Qys-main protease complex with PDB ID 6w2a and also reported the $\mathrm{IC}_{50}$ of Qys to SARS-CoV-2 is $0.45 \mu \mathrm{M}$, which is close to our prediction.

It is worth pointing out, except for the inhibitor T9j in the complex $5 \mathrm{rg} 1$, the rest of inhibitors reported in Table 1 are covalent inhibitors, which irreversibly form covalent bonds with Cys145 of the main protease (see discussion in Section 2.2.2). However, our models only predict the non-covalent binding affinity which is measured before the enzyme deactivation. The predicted binding affinities of all 137 complexes in SARS-CoV PBD-noBA dataset from various MathDL models are presented in Table S8 in ESI. $\dagger$ In this table, we also supply the synthetic accessibility score (SAS), partition coefficient $\log P$, and solubility $\log S$ for each small molecule. Except for SAS obtained via RDKit, ${ }^{13} \log P$ and $\log S$ are evaluated by our TopP-S model. ${ }^{14}$

\subsection{Discussion}

2.2.1 Binding site analysis. Based on the crystal structure information of 137 complexes in SARS-CoV PDB-noBA set, we have identified 13 distinct binding site regions of the SARS-CoV2 main protease as illustrated in Fig. 1. Those binding pockets are denoted by $\mathrm{P}_{i}, i=1,2, \ldots, 13$. Fig. 2 a reveals that binding pocket $\mathrm{P}_{1}$ is the most common binding region of the SARS-CoV2 main protease, which attracts around $80.2 \%$ of ligands in the SARS-CoV PDB-noBA data set of 137 complexes. This finding is no surprise since the binding pocket $\mathrm{P}_{1}$ shares similar active sites to its predecessor, i.e. SARS-CoV $\mathrm{M}^{\text {pro }}$. Specifically, $\mathrm{P}_{1}$ encompasses His141 and Cys145 catalytic dyad which are imperative to the substrate-binding mechanism. ${ }^{8}$ In additions, 

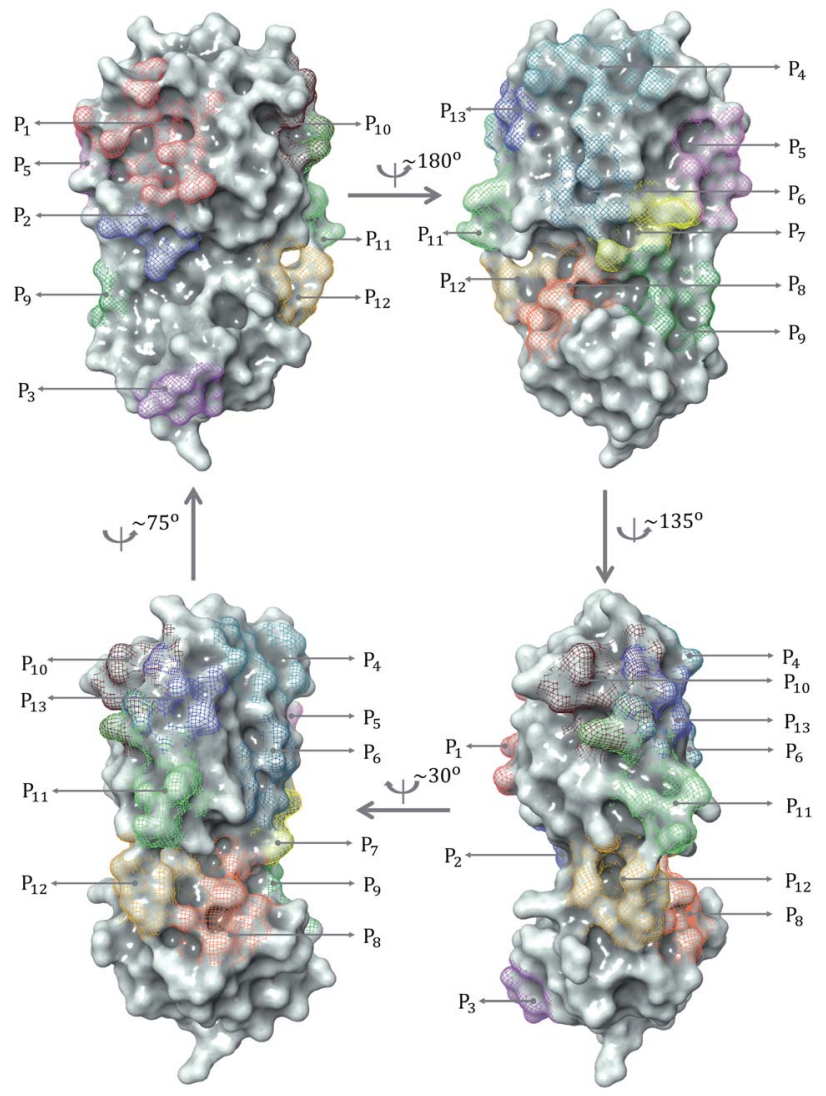

Fig. 1 All binding site pockets observed from 137 inhibitors in SARSCoV PDB-noBA set.

the substrate-binding residues Tyr161 and His163 (ref. 15) are covered in $\mathrm{P}_{1}$. Binding pockets $\mathrm{P}_{2}, \mathrm{P}_{3}, \mathrm{P}_{5}, \mathrm{P}_{7}, \mathrm{P}_{8}$, and $\mathrm{P}_{10}$ are the least favor sites consisting of only one ligand. The rest of the binding pockets involve no more than 7 ligands. To study the correlation of the binding regions to the binding free energy, we present the box plot in Fig. $2 \mathrm{~b}$ to illustrate the energy values through their quartiles.

The prevailing binding pocket $\mathrm{P}_{1}$ is the best region on the SARS-CoV-2 $\mathbf{M}^{\text {pro }}$ for inhibitor design with the median binding energy being $-7.22 \mathrm{kcal} \mathrm{mol}^{-1}$. Nol is the best inhibitor candidate for the binding site $\mathrm{P}_{1}$ with predicted affinity found to be $-8.90 \mathrm{kcal} \mathrm{mol}^{-1}$. Other binding regions such as $\mathrm{P}_{4}$, and $\mathrm{P}_{11}$ are less common but show their adequate effects on the binding mechanism with their best energy binding affinities calculated at $-7.28 \mathrm{kcal} \mathrm{mol}^{-1}$ and $-6.80 \mathrm{kcal} \mathrm{mol}^{-1}$, respectively. These potential binding sites can guide drug combination to inhibit coronavirus $\mathbf{M}^{\text {pro }}$ effectively.

2.2.2 Interaction analysis. By looking further into the interactions between the top inhibitors and the main protease, we have found that Nol, V2m, N3 are peptidomimetic inhibitors, they form as many as 8, 8, 9 hydrogen bonds respectively to the nearby residues and also all form 1 covalent bond with Cys145 as listed in Table 2 and depicted in Fig. 3. All these hydrogen bonds justify their potency of the first step of noncovalent binding to the main protease complex and confirms the robustness of our MathDL models; the covalent bonds make the binding irreversible. We also notice that these three inhibitors share two common hydrogen bonds to His163, His164 (see Table 2, Fig. 3a, c and d). Therefore, they have some similar predicted binding energies, especially $6 \mathrm{xhm}$ and 7bqy at $-8.50 \mathrm{kcal} \mathrm{mol}^{-1}$ and $-8.49 \mathrm{kcal} \mathrm{mol}^{-1}$, respectively.

This examination manifests how well our models preserve and capture the physical and chemical properties described in intermolecular bonding interactions. Furthermore, the ligand T9J that binds to $\mathbf{M}^{\text {pro }}$ in complex $5 \mathrm{rg} 1$ with a quite close binding energy at $-8.50 \mathrm{kcal} \mathrm{mol}^{-1}$ forms different hydrogen bonds in comparison to three previously mentioned inhibitors (see Table 2). Since our models only concern the non-covalent binding affinity, the lack of covalent bond in 5rg1's interactions does not downgrade its binding strength. With two relatively large hydrogen bonding distances (O2-His163: $3.05 \AA$, O3Glu166: $3.38 \AA$ (see Fig. 3d)), the binding affinity of 5rg1 is still comparable to the top inhibitors indicating the important roles in acquiring the hydrogen bonds to these residues in the main protease's binding process.

In the top 10 inhibitors as listed in Table 1, T9J in the complex $5 \mathrm{rg} 1$ is only one non-covalent inhibitor. The rest belongs to the class of targeted covalent inhibitors (TCI) in which they interacts with the protein residues, i.e., cysteine, to form a covalent complex strongly neutralizing target's function. However, the major disadvantage of TCIs is the association with the high toxicity risks. ${ }^{16}$ TCIs' strong covalent bond can irreversibly modify the unintended protein targets in the human body. As a result, the top covalent inhibitors in SARS-CoV PBDnoBA dataset may have little chance to become approved
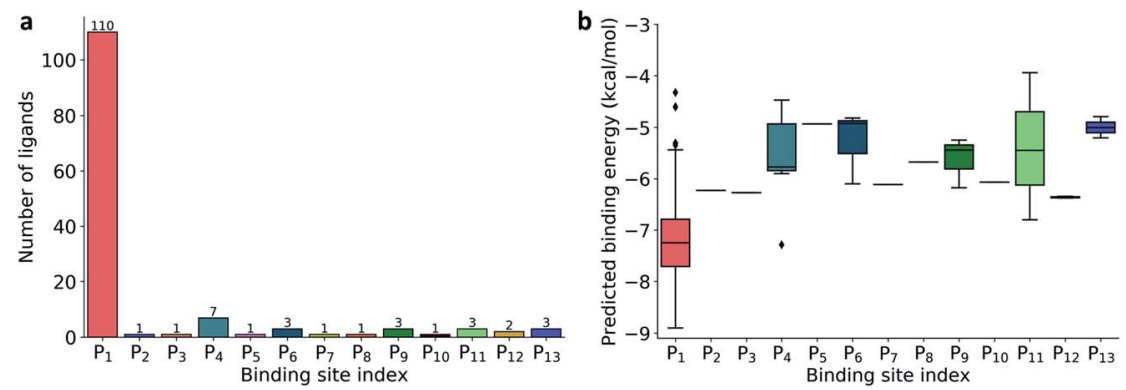

Fig. 2 (a) Distribution of 137 ligands across 13 distinct binding sites; (b) Box plot of predicted binding energies (kcal $\mathrm{mol}^{-1}$ ) of all inhibitors in each binding site. 
Table 2 Interaction analysis in the binding pockets of top 4 complexes in term of binding affinity predicted by our MathDL models

\begin{tabular}{lllr}
\hline PDB ID & Ligand ID & Hydrogen bond & Covalent bond \\
\hline 7c8t & Nol & His163, His164, Cys145, Gln189, Gly143, Glu166 & Cys145 \\
5rg1 & T9J & His163, Glu166 & Cys145 \\
6xhm & V2m & His163, His164, Cys145, Gln189, Phe140 & Cys145 \\
7bqy & N3 & His163, His164, Cys145, Gln189, Thr190, Glu166, Phe140, Gly143
\end{tabular}

market drugs in comparison to their non-covalent counterparts such as T9J in 5rg1.

Due to the popularity of the binding site $\mathrm{P}_{1}$ among 137 interested inhibitors, we mainly analyze the interaction network around the residues in that region. Out of 110 molecules binding to $\mathrm{P}_{1}$, there are 103 inhibitors forming at least one hydrogen bond to the nearby amino acid in the SARS-CoV-2 main protease. We have identified 20 different residues in the binding pocket $\mathrm{P}_{1}$ composing hydrogen bonds to these small molecules. Fig. 4 illustrates the frequency of these 20 residues across 110 inhibitors. Based on Fig. 4, Gly143 residue is the most attractive site to form the hydrogen bond. It appears in $53.6 \%$ of 110 intermolecular bonding interactions, followed by Glu166 residue with a frequency of $39.1 \%$; residue Cys145 and His163 also occupy $38.2 \%$ and $30.9 \%$, respectively. It is worth noting when these molecules form a hydrogen bond with Cys145, they also constitute another hydrogen bond with Gly143. In all cases, both these residues share the same
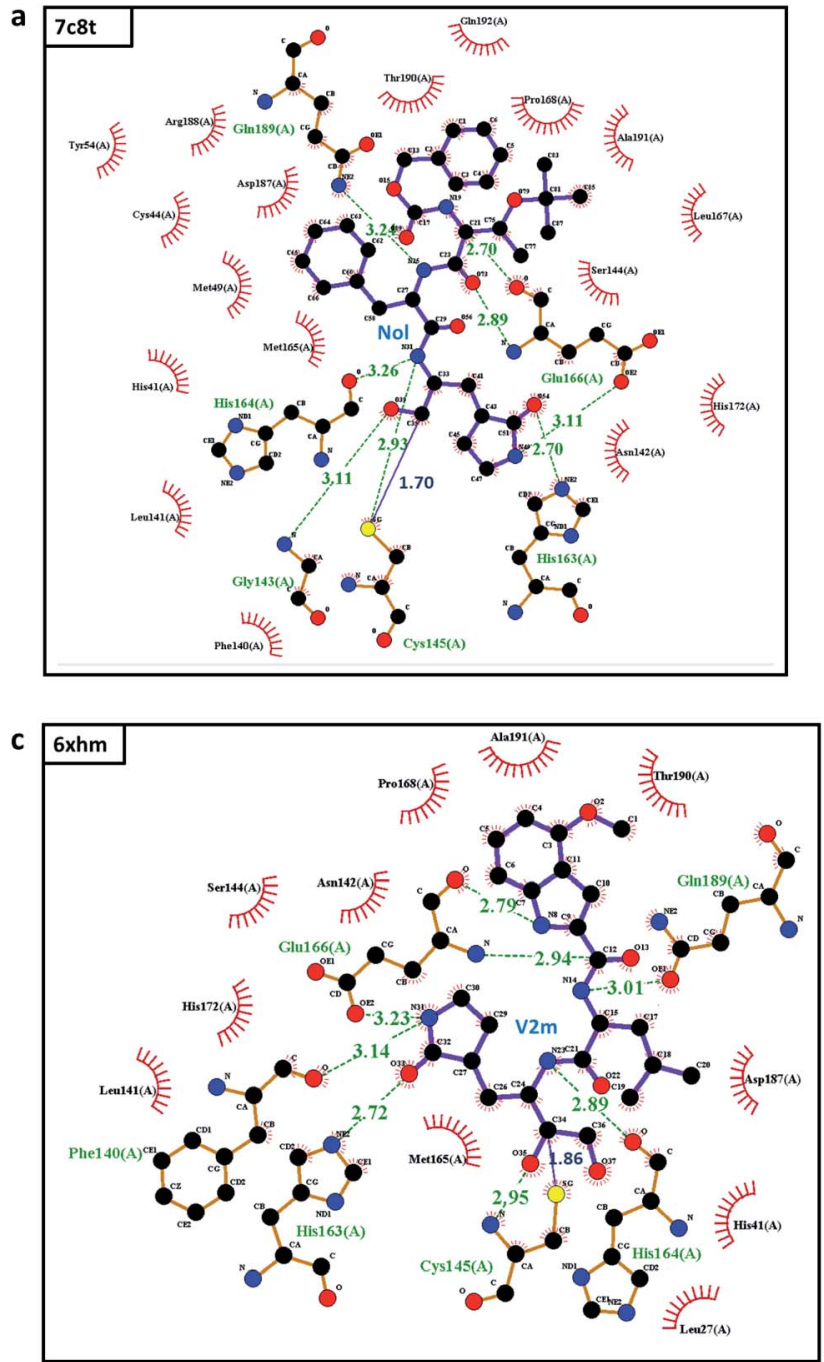
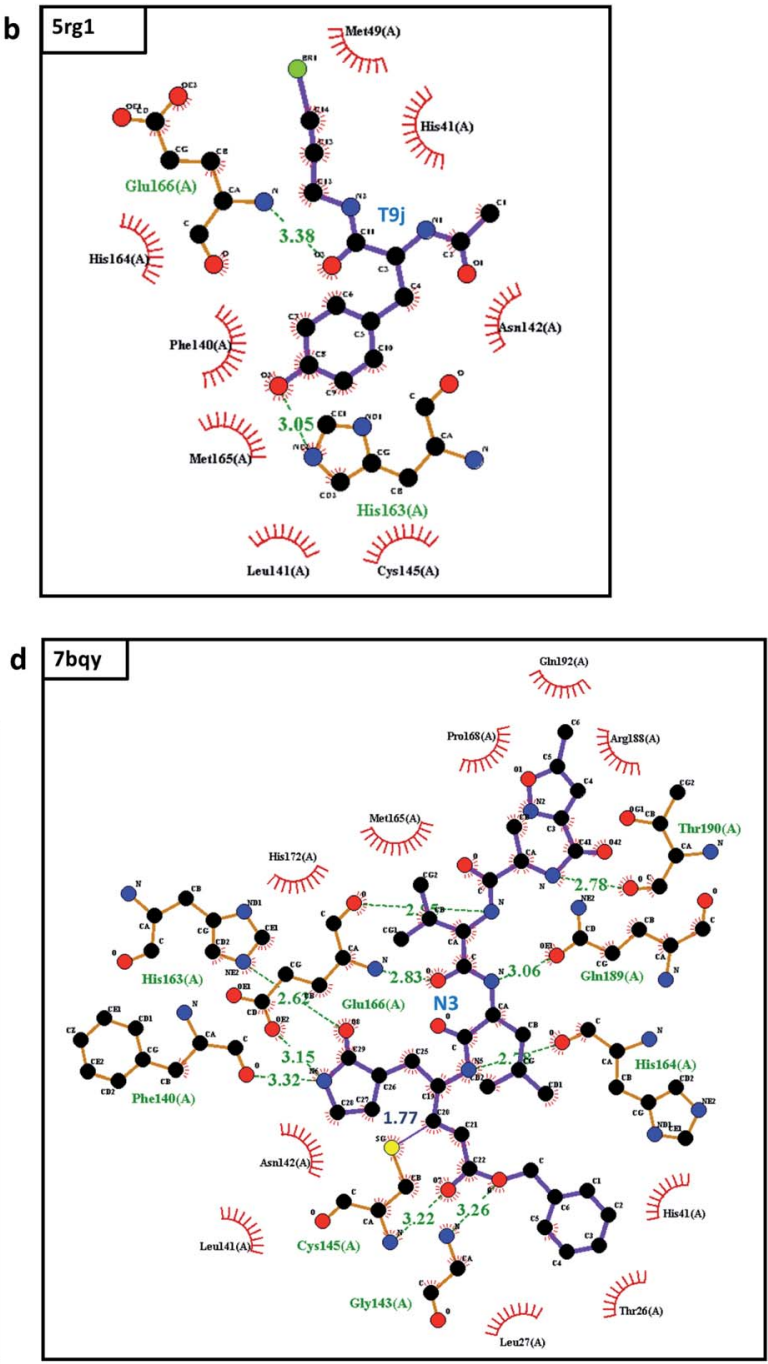

Fig. 3 The interactions between the top 4 inhibitors in the SARS-CoV PBD-noBA dataset and SARS-CoV-2 Mpro: (a) 7c8t; (b) 5rg1; (c) 6xhm; and (d) 7bqy. Inhibitors are shown in the purple color. Hydrogen bonds are marked in dashed green lines, and covalent bonds are depicted in solid blue lines. All interactions are shown with the distance information in $\AA$. 


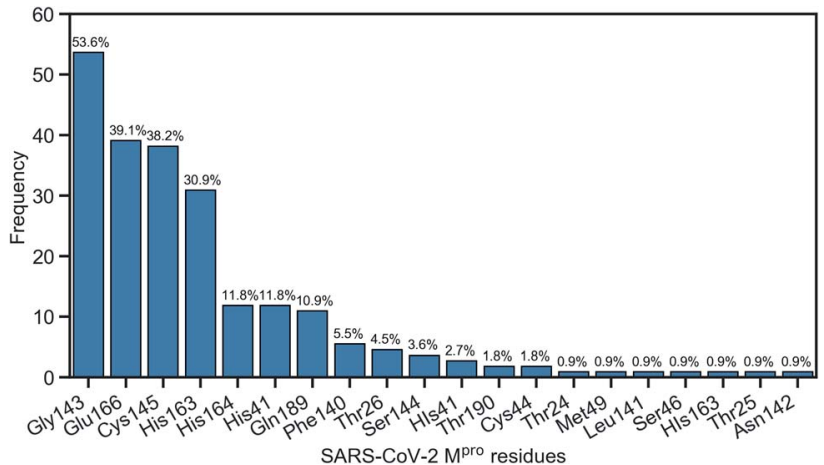

Fig. 4 Popularity of amino acids in the binding site $\mathrm{P}_{1}$ constituting the hydrogen bonds with ligands.

hydrogen-bond acceptor. Besides the hydrogen bond network, 71 ligands in the SARS-CoV PDB-noBA dataset form a covalent bond to $\gamma$-sulfur of Cys145. Except the second one, all the others in the top 10 inhibitors are equipped with that covalent bond (see Table S8 in ESI $\dagger$ ).

Furthermore, we are interested in the binding energy distribution associated with the interaction network. Fig. 5 depicts the violin plot of that distribution across four categories, namely no $\mathrm{H}$-bond (no hydrogen bond), H-bond (at least one hydrogen bond), no cov. bond (no covalent bond), and cov. bond (at least one covalent bond). Hydrogen bond interactions that are expected to play an important role in the binding mechanism are well captured in our MathDL models. Specifically, while the average energy of inhibitors having no hydrogen bond is $-6.62 \mathrm{kcal} \mathrm{mol}^{-1}$, the average energy of ones with hydrogen bond is as low as $-7.23 \mathrm{kcal} \mathrm{mol}^{-1}$.

It is noted that our MathDLs only measure the non-covalent binding affinity. The covalent bond appearing at the final covalent complex is not properly accounted for in our framework. Therefore, it is expected that our models sometimes overestimate the covalent-bond inhibitors over the noncovalent-bond candidates. Fig. 5 reveals molecules in the

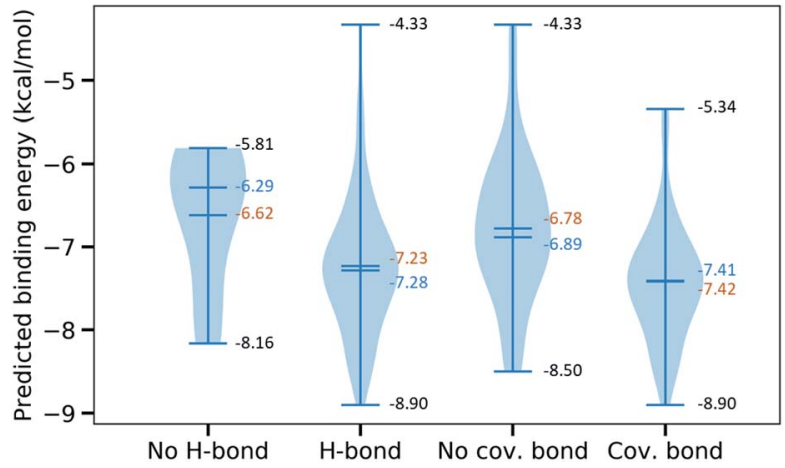

Fig. 5 Violin plot of the predicted binding energies for 110 inhibitors binding to the binding site $\mathrm{P}_{1}$ classified into 4 categories, namely no $\mathrm{H}$ bond (no hydrogen bond), $\mathrm{H}$-bond (at least one hydrogen bond), no cov. bond (no covalent bond), cov. bond (at least one covalent bond). The mean is in the orange color, the median is in the blue color, and the minimal and the maximal values are both in the black color. group of covalent bonds generally are predicted with lower binding energy with an average being $-7.42 \mathrm{kcal} \mathrm{mol}^{-1}$ in comparison to $-6.89 \mathrm{kcal} \mathrm{mol}^{-1}$ averagely measured on ones without covalent bonds.

2.2.3 Fragment analysis. To design the lead molecules, it is of importance to have promising fragments from existing inhibitors against the drug targets. Therefore, in the present work, we study all the fragments decomposed from 110 inhibitors attached to the binding site $\mathrm{P}_{1}$. To carry out this task, we utilize BRICS algorithm ${ }^{17}$ via RDkit. ${ }^{13}$ In BRICS model, there are 16 chemical environments indicated by linkers denoted by $L_{1}$, $\mathrm{L}_{2}, \ldots, \mathrm{L}_{16}$. The BRICS decomposition gives raise to a total of 185 unique fragments, which are all presented in Table S9 in ESI. $\dagger$ Fig. 6 illustrates top 12 common fragments in terms of their frequencies. Noting that the second frequent fragment, $\mathrm{L}_{1}$ $\mathrm{C}(\mathrm{C})=\mathrm{O}$, often constitutes a hydrogen bond with Gly143 and in many cases forms a covalent bond with Cys145.

\section{Materials and methods}

\subsection{Datasets}

Our deep learning-based scoring function, MathDL, was trained on public databases including PDBbind ${ }^{\mathbf{1 8}}$ and ChEMBL. ${ }^{4}$ The PDBbind sets contain all complexes with crystal structures deposited in the PDB with the binding affinities not limited to $K_{\mathrm{d}}, K_{\mathrm{i}}$, and $\mathrm{IC}_{50}$ reported in the literature. In this work, we employ the PDBbind v2019, the latest version of its generation. The v2019 version of the PDBbind consists of 17679 proteinligand complexes. However, the data preprocessing of the MathDL $^{32}$ only retains 17382 complexes. Among them, there are 10485 ligands measured in $K_{\mathrm{d}} / K_{\mathrm{i}}$ and 6537 ligands measured in $\mathrm{IC}_{50}$.

ChEMBL is another manually curated database of bioactive molecules. Currently, ChEMBL contains more than 2 million compounds in the SMILES string format. Excluding 30 main protease inhibitors in PDBbind data, we have found other 277 small molecules on ChEMBL with reported $K_{\mathrm{d}} / \mathrm{IC}_{50}$. Additionally, we have found more than 300 other SARS-CoV main protease inhibitors from literatures. ${ }^{\mathbf{1 8 - 2 0 , 2 5 - 3 1}}$ In total, there are more than 600 ligands bound to SARS-CoV/SARS-CoV-2 main protease having the experimental binding affinities; among them, there are 44 crystal structures. For compounds without the crystal structures, MathPose ${ }^{6}$ is utilized to generate their 3D conformations. The predicted 3D coordinates of these structures are presented in the SDF format and available in ESI. $\dagger$ Currently, there are roughly 137 ligands forming crystal complexes with the SARS-CoV-2 main protease on PDB without the report of the experimental inhibitor activities. Most of them are deposited by the PanDDA analysis group (https:// pandda.bitbucket.io/\#).

To serve model validation purposes, we classify the selected data into five different groups as listed in Table 3. Specifically, PDBbind v2019 is the biggest set in this compilation with its PDB IDs and experimental binding affinities listed in Table S1 in ESI. $\dagger$ PDBbind v2016 core set is a subset of PDBbind v2019 and is formed by 290 complexes representing all protein classes in the refined set of PDBbind v2016. ${ }^{18,33}$ The PDB IDs of all 
<smiles>CN[AsH2]</smiles>

Frequency: 70<smiles>[3H]C([13CH3])=O</smiles>

Frequency: 23<smiles>[3H]C(C)=O</smiles>

Frequency: 53<smiles>[B]CI</smiles>

Frequency: 33<smiles>[3H]C([3H])=O</smiles>

Frequency: 18<smiles>[AsH2]N1CCN([AsH3])CC1</smiles>

Frequency: 24<smiles>CO[123I]OC</smiles>

Frequency: 16<smiles>[3H]C(=O)[C@H](CC)CC(C)C</smiles>

Frequency: 12<smiles>CC(F)(S)[C@H]1CCNC1=O</smiles>

Frequency: 12<smiles>[Te]c1cccnc1</smiles>

Frequency: 12<smiles>[12CH3]C(=O)C[13CH3]</smiles>

Frequency: 11

Fig. 6 Fragment frequencies based on BRICS decomposition of 110 inhibitors of binding site pocket $P_{1}$. $L_{i}$ is the link atom of a certain type described in ref. 17.

Table 3 A summary of our selected data sets

\begin{tabular}{|c|c|c|c|}
\hline Data name & Data size & Descriptions & References \\
\hline PDBbind v2019 & 17382 & Partial PDBbind general set v2019 & 18 \\
\hline PDBbind v2016 core set & 290 & PDBbind v2016 core set & 18 \\
\hline SARS-CoV PDB-BA & 44 & $\begin{array}{l}\text { Inhibitors of SARS-CoV/SARS-CoV-2 } \\
\mathbf{M}^{\text {pro }} \text { having X-ray crystal structures } \\
\text { and experimental binding affinities }\end{array}$ & $5,18-23$ \\
\hline SARS-CoV 2D & 141 & $\begin{array}{l}\text { Inhibitors of SARS-CoV/SARS-CoV-2 } \\
\mathrm{M}^{\text {pro }} \text { having only } 2 \mathrm{D} \text { structures }\end{array}$ & $4,19,20,25-31$ \\
\hline SARS-CoV BA & 185 & $\begin{array}{l}\text { Inhibitors of SARS-CoV/SARS-CoV-2 } \\
\mathbf{M}^{\text {pro }} \text { having experimental binding } \\
\text { affinities }\end{array}$ & $5,18-20,26-31$ \\
\hline
\end{tabular}

complexes in the PDBbind v2016 core set are provided in Table S2. $\dagger$ We also collect all $\mathrm{M}^{\text {pro }}$ complexes of SARS-CoV/SARS-CoV-2 on the PDB, denoted by SARS-CoV PDB, which results in a total of 192 structures (see Table S3†). Among them, there are 44 ligands with the report of experimental binding affinities denoted by SARS-CoV PDB-BA (see Table S4 $\dagger$ ). Furthermore, we are interested in the set of SARS-CoV- $2 \mathbf{M}^{\text {pro }}$ complexes in the aforementioned SARS-CoV PDB set but their affinities are not presented or undisclosed. We call this set SARS-CoV PDB-noBA with PDB IDs listed in Table S5. $\uparrow$ To enrich our training data targeting SARS-CoV/SARS-CoV-2 main protease inhibitors, we gather some inhibitors reported on the literature. ${ }^{4,25}$ For those compounds with only 2D information, we limit ourselves to ones having the similarity score based on the path-based fingerprint FP2 no lower than 0.6 to at least one inhibitor in the SARS-CoV PDB set. As a result, we arrive at a set of 141 structures named SARS-CoV 2D (see Table S6†). Combining SARS-CoV PDB-BA and SARS-CoV 2D data sets, we finalize a reliable database focusing on SARS-CoV/SARS-CoV-2 main protease inhibitors. Notice that the binding affinities in this set are all reported in $\mathrm{IC}_{50}$. Table $\mathrm{S} 7$ in ESI $\uparrow$ presents the PDB IDs as well as the experimental binding energies of these ligands.

\subsection{Methods}

3.2.1 MathDL. The MathDL models developed in this work are reformulated from our early model bearing the same name. MathDL was designed for the prediction of various druggable properties of 3D molecules. ${ }^{6}$ In the past three years, MathDL has been proved to be the top competitor in D3R Grand Challenges 

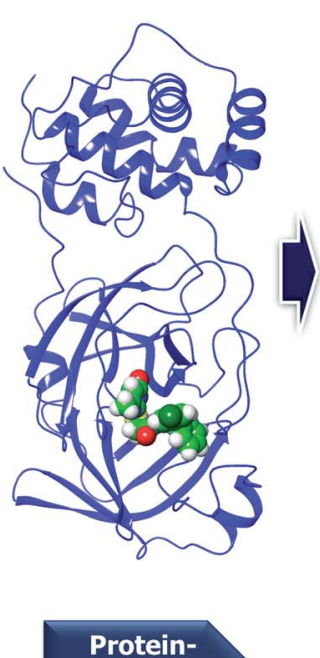

ligand

complex
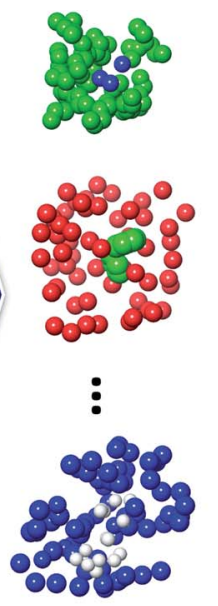

Element

specific

groups

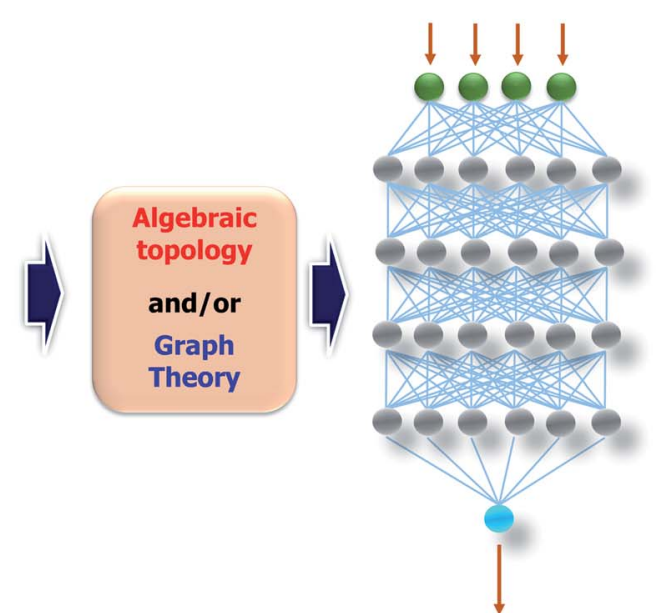

Mathematical representations
Machine

learning

prediction

Fig. 7 A framework of MathDL energy prediction model which integrates advanced mathematical representations with sophisticated CNN architectures.

(https://drugdesigndata.org/about/grand-challenge), a worldwide competition in computer-aided drug design. In the present work, we have, for the first time, develop a multitask MathDL (MathDL-MT) to handle the $\mathrm{M}^{\text {pro }}$ inhibitor dataset. We have also extended our earlier MathDL by including all different datasets (MathDL-All). Fig. 7 depicts the framework of the MathDL in which the element-specific algebraic topological representations are integrated with the convolutional neural network (CNN) aiming to predict varied druggable properties such as toxicity, binding affinities, etc.

3.2.1.1 Algebraic topology-based representations. Algebraic topology studies the topological spaces with the use of abstract algebra, which can dramatically simplify the geometric complexity. Persistent homology ( $\mathrm{PH})$ is one of the algebraic topology approaches which has the capacity to track the multiscale topological information over different scales along with filtration by characterizing independent components, rings, and higher dimensional voids in space. ${ }^{34}$ In this section, we will briefly review the algebraic topology-based representations. Additionally, since we are dealing with the protein-ligand system, therefore, the biological considerations will take into account as well.

Simplex. The $q$-simplex denoted as $\sigma_{q}$ is the convex hull of $q+$ 1 affinely independent points in $\mathbb{R}^{n}(n \geq k)$. For example, the 0 , 1,2 , and 3-simplex is considered as a vertex, an edge, a triangle, and a tetrahedron, respectively. We call the convex hull of each non-empty subset of $q+1$ points the face of $\sigma_{q}$, and each points are also called the vertices.

Simplicial complex. A set of simplices is a simplicial complex denote $K$ which satisfies that every face of a simplex $\sigma_{q} \in K$ is also in $K$ and the non-empty intersection of any two simplices in $K$ is the common face for both.
Chain complex. A formal sum of $q$-simplices in simplicial complex $K$ with coefficients in an algebraic field (typically $\mathbb{Z}_{2}$ ) is a $q$-chain. A set of all $q$-chains of the simplicial complex $K$ equipped with an algebraic field is called a chain group and denoted as $C_{q}(K)$. The boundary operator is defined by $\partial_{q}: C_{q}(K)$ $\rightarrow C_{q-1}(K)$ to relate the chain groups. More specifically, we denote $\sigma_{q}=\left[v_{0}, v_{1}, \ldots, v_{q}\right]$ for the $q$-simplex spanned by its vertices, and then the boundary operator can be represented as:

$$
\partial_{q} \sigma_{q}=\sum_{i=0}^{q}(-1)^{i} \sigma_{q-1}^{i} .
$$

Here, $\sigma_{q-1}{ }^{i}=\left[v_{0}, \ldots, \hat{v}_{i}, \ldots, v_{q}\right]$ is the $(q-1)$-simplex with $v_{\mathrm{i}}$ being omitted. The sequence of chain groups connected by boundary operators is called the chain complex and expressed as:

$$
\cdots \stackrel{\partial_{q+2}}{\longrightarrow} C_{q+1}(K) \stackrel{\partial_{q+1}}{\longrightarrow} C_{q}(K) \stackrel{\partial_{q}}{\rightarrow} C_{q-1}(K) \stackrel{\partial_{q-1}}{\longrightarrow} \cdots
$$

The $q$-cycle group $Z_{q}(K)$ and the $q$-boundary group $B_{q}(K)$ are defined as $Z_{q}(K)=\operatorname{ker}\left(\partial_{q}\right)=\left\{c \in C_{q}(K) \mid \partial_{q} c=\varnothing\right\}$ and $B_{q}(K)=$ $\operatorname{im}\left(\partial_{q+1}\right)=\left\{\partial_{q+1} c \mid c \in C_{q+1}(K)\right\}$. The $q$-th homology group is the quotient group $H_{q}(K)=Z_{q}(K) / B_{q}(K)$. Moreover, the rank of $q$-th homology group can be computed as $\operatorname{rank} H_{q}(K)=\operatorname{rank} Z_{q}(K)-$ $\operatorname{rank} B_{q}(K)$, which is denoted as the $q$-th Betti number $\beta_{q}$. To be notice that the $q$-th Betti number count the number of $q$ dimensional holes that can not be continuously deformed to each other.

Persistent homology. A filtration of a simplicial complex $K$ is a nested sequence of subcomplexes of $K$ such that $\varnothing=K_{0} \subseteq K_{1}$ $\subseteq K_{2} \cdots \subseteq K_{m}=K$. Then the $p$-persistent $q$ th homology group of $K_{t}$ is defined as:

$$
H_{q}^{p}\left(K_{t}\right)=Z_{q}\left(K_{t}\right) /\left(B_{q}\left(K_{t+p}\right) \cap Z_{q}\left(K_{t}\right)\right)
$$


Here the rank of $H_{q}^{p}\left(K_{t}\right)$ counts the number of $q$-dimensional holes in $K_{t}$ that are still alive in $K_{t+p}$, which is called the $p$ persistent $q$ th Betti number. The persistent homology not only records the topological information at a specific configuration, but also tracks the changes along with the filtration parameters. More specifically, the topological changes will be preserved in the persistent barcodes. In MathDL, we make use of the persistent homology barcodes by dividing them into bins and calculating the birth, death, and persistence incidents in each bin to enrich our algebraic topological representations.

3.2.1.2 Element specific considerations. The protein-ligand complex is structural and also biological. The persistent homology provides a theoretical approach to encode highdimensional spatial data of protein-ligand complexes into algebraic topological representations. In this section, we address the biological considerations for biomolecular complexity. There are many kinds of interactions that exist in the protein-ligand complex, such as electrostatics, hydrogen bonds, and hydrophobic effects. Although persistent homology can capture the interactions among the nearest neighbors, the long-range interactions will be hindered. This difficulty can be avoided via the deployment of the element-specific attention. ${ }^{32}$ There are 4 commonly atom types in protein, namely $\mathrm{C}, \mathrm{N}, \mathrm{O}, \mathrm{S}$, and there are 11 commonly atom types in ligand, including $\mathrm{C}, \mathrm{N}, \mathrm{O}, \mathrm{S}, \mathrm{P}, \mathrm{F}, \mathrm{Cl}, \mathrm{Br}, \mathrm{I}, \mathrm{H}, \mathrm{B}$. We include Boron in the ligand atom type consideration since it appears in more than 200 small compounds in our training data. The general framework of MathDL is depicted in Fig. 7 under exemplified steps. In addition, the details of the deep learning architecture of the current MathDL is offered in Fig. S1. $\dagger$ For the details of feature descriptions as well as the deep learning architecture, interested readers are referred to our previous work. ${ }^{32}$

3.2.2 MathPose. MathPose, a 3D pose predictor that converts SMILES strings into 3D poses with references of target molecules, is the top performer in D3R Grand Challenge 4 (GC4) in predicting the poses of 24 beta-secretase 1 (BACE) binders. ${ }^{6}$ For one SMILES string, around 1000 3D conformations can be generated by various docking software tools such as GOLD, ${ }^{35}$ Autodock Vina, ${ }^{36}$ and GLIDE. ${ }^{37}$ Moreover, a selected set of known complexes is re-docked by three aforementioned docking software packages to generate at least 100 decoy complexes per input ligand used in the machine learning training set. The machine learning labels will be the calculated root mean squared deviations (RMSDs) between the decoy and native structures for the training data of the pose selection task. Furthermore, MathDL models will be set up and applied to select the top-ranked pose for the given ligand. Besides the GC4 challenge, our models have outperformed state-of-the-art scoring functions at the docking power challenge on CASF2007 and CASF-2013 benchmarks. ${ }^{33}$ Those established results attest to the credibility of our MathPose on the 3D structure prediction of small molecules.

\subsection{Validations}

3.3.1 PDBbind v2016 core set benchmark. In this validation task, we will testify our model against 290 complexes in the
PDBbind v2016 core set. This is a prevalent test set to assert the scoring ability of a binding affinity prediction model and has attracted lots of research groups to devote the effort to improve the Pearson's correlation coefficient $\left(R_{\mathrm{p}}\right)$ and Kendall's tau $(\tau)$ on this core set performance. ${ }^{\mathbf{1 8 , 4 2 , 4 3}}$ In the current work, we merge the PDBbind v2019, SARS-CoV PDB-BA, and SARS-CoV 2D sets but removing the duplicates and excluding the PDBbind v2016 core set complexes to attain a training set of 17211 complexes. MathDL with the architecture described in Section 3.2.1 is trained on those complexes. The resulting model is utilized to predict the binding affinity of 290 structures in the PDBbind v2016 core set.

With the purpose of exploring the most optimal model for this benchmark, MathDL is trained for 1000 epochs. Then, we pick the epoch based on the root-mean-squared error (RMSE) of the PDBbind v2016 core set prediction. We have found that MathDL achieves the smallest RMSE in this experiment at 140 epochs. Specifically RMSE, $R_{\mathrm{p}}$, and $\tau$ metrics on the v2016 core set are $1.56 \mathrm{kcal} \mathrm{mol}^{-1}, 0.858$, and 0.671 , respectively. Meanwhile, the training accuracy is $0.387 \mathrm{kcal} \mathrm{mol}^{-1}$ in terms of RMSE and its Pearson's correlation coefficient is $R_{\mathrm{p}}=0.994$. These performances reveal that our MathDL converges very fast and with only 140 epochs and maintains a good balance between training and testing accuracies. This is a state-of-theart performance since our MathDL is ranked in the second place in comparison to 33 other scoring functions (see Fig. 8). It is noted that the top model is $\mathrm{TopBP}_{\text {con. }}$ published in our previous work ${ }^{32}$ with $R_{\mathrm{p}}=0.861$. TopBP $\mathrm{P}_{\text {con. }}$ is the consensus of gradient boosted tree and deep learning-based models. If only the deep learning framework is considered, the performance of TopBP (denoted by TopBP-DL) on the core set of PDBbind v2016 is $R_{\mathrm{p}}=0.848$.

It is worth mentioning that except for our MathDL, all machine learning-based scoring functions listed in Fig. 8 were trained on the PDBbind v2016 refined set of 3767 complexes. As mentioned above, the current MathDL is compiled on a much larger training set comprised of 17211 complexes selected from PDBbind v2019 and SARS-CoV BA data. Even the present MathDL has not outperformed its predecessor, i.e., TopBP $\mathrm{P}_{\text {con. }}$, MathDL is still a preference model since it is trained on a diverse data set covering various protein families and different binding energy ranges. As a result, it is expected to deliver more reliable predictions on the SARS-CoV-2 inhibitor, especially when this main protease family is not included in the training data of previous TopDL models. The resulting MathDL model is labeled as MathDL-Core2016 and is utilized to predict affinities of complexes in SARS-CoV PDB-noBA in Section 2.1.

3.3.2 5 fold cross-validation on SARS-CoV BA set. In this section, we testify the performance of our MathDL against 185 inhibitors in the SARS-CoV BA set aforementioned in Table 3. Among those ligands, there are 44 X-ray crystal structures and the rest are in 2D SMILES strings. We employ MathPose to predict $3 \mathrm{D}$ structures of those $2 \mathrm{D}$ ligands. To carry out the validation, we randomly split the SARS-CoV BA set into 5 nonoverlapped folds. In each fold prediction task, MathDL trains on the partial data of SARS-CoV BA in conjunction with PDBbind v2019 set. This situation results in two different ways 


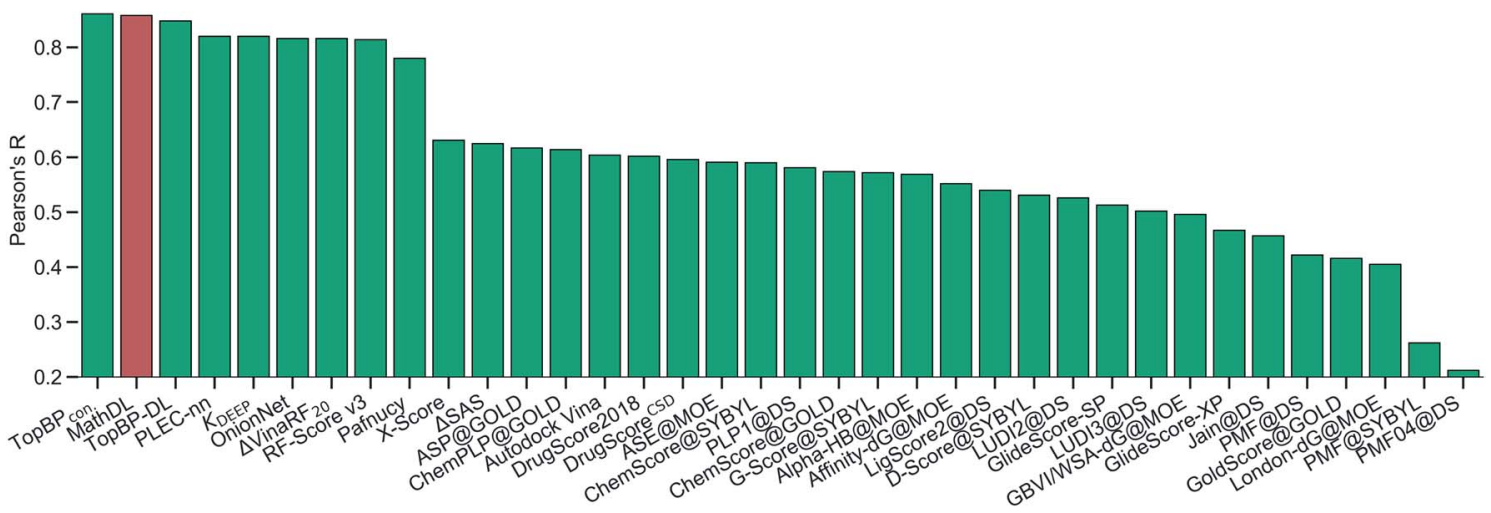

Fig. 8 The Pearson correlation coefficient of various scoring functions on PDBbind v2016 core set benchmark. Our MathDL is in red. The

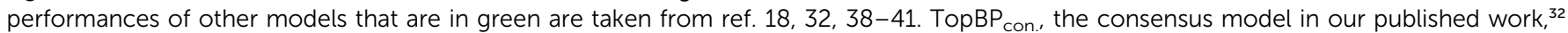
attains the highest $R_{\mathrm{p}}$ at 0.861 . The current MathDL is followed with the second highest $R_{\mathrm{p}}$ at 0.858 and RMSE $=1.56 \mathrm{kcal}^{\mathrm{mol}}{ }^{-1}$. The third place in the list is another TopBP model, TopBP-DL, solely based on the deep learning architectures and its reported $R_{\mathrm{p}}$ is 0.848 . $^{32}$ It is noted that all of the machine learning based scoring functions in this comparison were trained on the PDBbind v2016 refined set of 3767 complexes except for our MathDL. Explicitly, MathDL is trained on a much larger training set consisting of 17211 complexes picked out from the PDBbind v2019 set and SARS-COV BA set.

of training our MathDL model. The first approach is a traditional MathDL architecture with the training set combining both SARS-CoV BA and PDBbind v2019 complexes. The second model makes use of multi-task learning. ${ }^{44}$ In each epoch, the weights of the MathDL architecture are learned through the information from PDBbind v2019 set, then only the fully connected layers are trainable when learning SARS-CoV BA structures. Finally, we come up with 10 different MathDL models in which the traditional MathDL frameworks are labeled as MathDL-All- $i$ and multi-task MatDL is named MathDL-MT- $i$ with $i$ running from 1 to 5 . In each model, after 100 epochs, we start monitoring which epoch that helps our model achieve the smallest RMSE on the test set.

Table 4 reveals that MathDL-All models are well trained with the averaged accuracy RMSE $=0.286 \mathrm{kcal} \mathrm{mol}^{-1}$, Pearson's correlation coefficient $R_{\mathrm{p}}=0.994$, and Kendall's tau $\tau=0.934$. Their averaged performances on test data across 5-fold of the SARS-CoV BA set are found to be $R_{\mathrm{p}}=0.729, \tau=0.540$, and

Table 4 5-fold Performances of MathDL-All and MathDL-MT on SARS-COV BA set

\begin{tabular}{lllllllll}
\hline & \multicolumn{3}{l}{ MathDL-ALL } & & & \multicolumn{2}{l}{ MathDL-MT } \\
\cline { 2 - 3 } & $R_{\mathrm{p}}$ & $\tau$ & RMSE & & $R_{\mathrm{p}}$ & $\tau$ & \\
\hline Fold 1 (train) & 0.992 & 0.923 & 0.327 & & 0.996 & 0.949 & 0.253 \\
Fold 1 (test) & 0.792 & 0.534 & 0.682 & & 0.818 & 0.534 & 0.680 \\
Fold 2 (train) & 0.995 & 0.943 & 0.266 & & 0.996 & 9.948 & 0.236 \\
Fold 2 (test) & 0.625 & 0.498 & 0.866 & & 0.689 & 0.538 & 0.826 \\
Fold 3 (train) & 0.991 & 0.917 & 0.367 & & 0.994 & 0.934 & 0.327 \\
Fold 3 (test) & 0.771 & 0.572 & 0.758 & & 0.767 & 0.593 & 0.802 \\
Fold 4 (train) & 0.996 & 0.948 & 0.240 & & 0.997 & 0.951 & 0.177 \\
Fold 4 (test) & 0.618 & 0.397 & 0.874 & & 0.642 & 0.472 & 0.901 \\
Fold 5 (train) & 0.995 & 0.941 & 0.231 & & 0.991 & 0.921 & 0.380 \\
Fold 5 (test) & 0.838 & 0.699 & 0.767 & & 0.719 & 0.524 & 0.900 \\
Average (train) & 0.994 & 0.934 & 0.286 & & 0.995 & 0.941 & 0.275 \\
Average (test) & 0.729 & 0.540 & 0.789 & & 0.727 & 0.532 & 0.822
\end{tabular}

RMSE $=0.789 \mathrm{kcal} \mathrm{mol}^{-1}$. These results endorse the reliability of these models in the binding affinity prediction of SARS-CoV/ SARS-CoV-2 inhibitors. Table 4 also lists the training and testing performances of five multi-task learning models. The averaged training performance of the MathDL-MT model is $R_{\mathrm{p}}=0.995, \tau$ $=0.941$ and RMSE $=0.275 \mathrm{kcal} \mathrm{mol}^{-1}$. The accuracy of the multi-task architecture on the test sets is similar to MathDL-All with $R_{\mathrm{p}}=0.727, \tau=0.532$, and $\mathrm{RMSE}=0.822 \mathrm{kcal} \mathrm{mol}^{-1}$. With these promising results, it is encouraging to carry out MathDL models to predict unknown binding affinities of SARS-CoV/ SARS-CoV-2 inhibitors. It is worth noting that if the 5-fold cross-validation is conducted purely on the SARS-CoV BA set, the average $R_{\mathrm{p}}$ and $\tau$ are as low as 0.561 and 0.388 , respectively. These results strongly support the inclusion of diverse information such as PDBbind v2019 in conjunction with sophisticated deep learning architectures to achieve the accurate binding energy prediction of $\mathrm{M}^{\text {pro }}$ inhibitors.

\section{Conclusion}

SARS-CoV-2 main protease $\left(\mathrm{M}^{\mathrm{pro}}\right)$ is the most favorable target for COVID-19 drug discovery due to its conservative nature and low similarity with human genes. Structure and binding affinity of protein-drug complexes are of paramount importance for understanding the molecular mechanism in drug discovery. However, there are only two SARS-CoV-2 $\mathrm{M}^{\text {pro }}$ inhibitor structures available with binding affinities, highlighting current challenges in COVID-19 drug discovery.

This work presents the reliable binding affinity prediction and ranking of $137 \mathrm{M}^{\text {pro }}$-inhibitor crystal structures that have no reported experimental binding affinity. We first curate a set of more than $600 \mathrm{M}^{\text {pro }}$ inhibitors with binding affinities from public resources, such as PDBbind, ChEMBL and the scattered literature. Among these inhibitors, 141 are retained based on their high similarity with available $\mathrm{M}^{\text {pro }}$-inhibitor complex structures and built with three dimensional (3D) poses using 
our MathPose. ${ }^{6}$ Together with 44 another SARS-CoV or SARS-

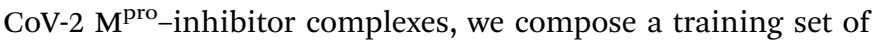
185 reliable SARS-CoV-2 $\mathbf{M}^{\text {pro }}$-inhibitor complexes. Our earlier MathDL models are reformulated with algebraic topology to accommodate 119 new complexes and 17382 complexes from the PDBbind v2019 general set in both single-task and multitask settings, which have never been available before. The resulting MathDL models are rigorously validated via PDBbind v2016 core set benchmark in which it outperforms state-of-the-art models in the literature. Most importantly, our MathDL achieves promising cross-validation accuracies on the SARS-CoV family inhibitors with the averaged Pearson's correlation coefficient as high as 0.73 .

Additionally, the present work unveils that Gly143 of $\mathrm{M}^{\text {pro }}$ is the most attractive region to form hydrogen bonds, followed by Glu166, Cys145, and His163. There are 71 inhibitors interacting with SARS-CoV-2 $\mathrm{M}^{\text {pro }}$ to form covalent complexes. Those covalent bonds are mostly composed between dicarbon monoxide groups in inhibitors and $\gamma$-sulfur on Cys145. There are only one non-covalent complex in our top 10 ranked, namely 5rg1. To provide a potential resource for lead molecule design, we employ the BRICS algorithm to decompose all the inhibitors of the prominent binding site on $\mathrm{M}^{\mathrm{pro}}$ and obtain 185 unique fragments.

The predicted binding affinities and their ranking of 137

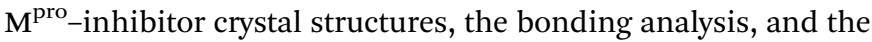
fragment decomposition have significantly extended current knowledge and understanding of SARS-CoV-2 $\mathrm{M}^{\text {pro }}$ and inhibitor interactions and, thus offered valuable information toward COVID-19 drug discovery.

\section{Conflicts of interest}

There are no conflicts to declare.

\section{Acknowledgements}

This work was supported in part by NIH grant GM126189, NSF Grants DMS-1721024, DMS-1761320, and IIS1900473, Michigan Economic Development Corporation, George Mason University award PD45722, Bristol-Myers Squibb, and Pfizer. The authors thank The IBM TJ Watson Research Center, The COVID-19 High Performance Computing Consortium, and NVIDIA for computational assistance.

\section{References}

1 Phase III Double-blind, Placebo-controlled Study of AZD1222 for the Prevention of COVID-19 in Adults, 2020, accessed September 15, 2020, https://clinicaltrials.gov/ct2/show/ NCT04516746?term $=$ NCT04516746\&draw $=2 \&$ rank $=1$.

2 Statement on AstraZeneca Oxford SARS-CoV-2 vaccine, AZD1222, COVID-19 vaccine trials temporary pause, 2020, accessed September 15, 2020, https:/www.astrazeneca.com/ media-centre/press-releases/2020/statement-on-astrazenecaoxford-sars-cov-2-vaccine-azd1222-covid-19-vaccine-trialstemporary-pause.html.
$3 \mathrm{X} . \mathrm{Xu}, \mathrm{P}$. Chen, J. Wang, J. Feng, H. Zhou, X. Li, Wu Zhong and P. Hao, Evolution of the novel coronavirus from the ongoing wuhan outbreak and modeling of its spike protein for risk of human transmission, Sci. China: Life Sci., 2020, 63(3), 457-460.

4 M. Davies, M. Nowotka, G. Papadatos, N. Dedman, A. Gaulton, F. Atkinson, L. Bellis and J. P. Overington, Chembl web services: streamlining access to drug discovery data and utilities, Nucleic Acids Res., 2015, 43(W1), W612W620.

5 H. M. Berman, J. Westbrook, Z. Feng, G. Gilliland, T. N. Bhat, H. Weissig, I. N. Shindyalov and P. E. Bourne, The protein data bank, Nucleic Acids Res., 2000, 28(1), 35-242.

6 D. D. Nguyen, K. Gao, M. Wang and G.-W. Wei, MathDL: Mathematical deep learning for $\mathrm{d} 3 \mathrm{r}$ grand challenge $4, \mathrm{~J}$. Comput.-Aided Mol. Des., 2020, 34, 131-147.

7 D. A. Matthews, P. S. Dragovich, S. E. Webber, S. A. Fuhrman, A. K. Patick, L. S. Zalman, T. F. Hendrickson, R. A. Love, T. J. Prins, J. T. Marakovits, et al., Structure-assisted design of mechanism-based irreversible inhibitors of human rhinovirus $3 \mathrm{c}$ protease with potent antiviral activity against multiple rhinovirus serotypes, Proc. Natl. Acad. Sci. U. S. A., 1999, 96(20), 11000-11007.

8 H. Yang, W. Xie, X. Xue, K. Yang, J. Ma, W. Liang, Q. Zhao, Z. Zhou, D. Pei, J. Ziebuhr, et al., Design of wide-spectrum inhibitors targeting coronavirus main proteases, PLoS Biol., 2005, 3(10), e324.

9 S. Yang, S.-J. Chen, M.-F. Hsu, J.-D. Wu, C.-T. K. Tseng, Y.-F. Liu, H.-C. Chen, C.-W. Kuo, C.-S. Wu, L.-W. Chang, et al., Synthesis, crystal structure, structure-activity relationships, and antiviral activity of a potent sars coronavirus $3 \mathrm{cl}$ protease inhibitor, J. Med. Chem., 2006, 49(16), 4971-4980.

10 F. Wang, C. Chen, W. Tan, K. Yang and H. Yang, Structure of main protease from human coronavirus nl63: insights for wide spectrum anti-coronavirus drug design, Sci. Rep., 2016, 6, 22677.

11 Z. Jin, X. Du, Y. Xu, Y. Deng, M. Liu, Y. Zhao, B. Zhang, X. Li, L. Zhang, C. Peng, et al., Structure of mpro from covid-19 virus and discovery of its inhibitors, bioRxiv, 2020.

12 A. D. Rathnayake, J. Zheng, Y. Kim, K. D. Perera, S. Mackin, D. K. Meyerholz, M. M. Kashipathy, K. P. Battaile, S. Lovell, S. Perlman, et al., 3c-like protease inhibitors block coronavirus replication in vitro and improve survival in mers-cov-infected mice, Sci. Transl. Med., 2020, 12(557), eabc5332.

13 G. Landrumet al., Rdkit: Open-source cheminformatics, 2006.

14 K. Wu, Z. Zhao, R. Wang and G. W. Wei, TopP-S: Persistent Homology-Based Multi-Task Deep Neural Networks for Simultaneous Predictions of Partition Coefficient and Aqueous Solubility, J. Comput. Chem., 2018, 39, 1444-1454.

15 H.-P. Chang, C.-Y. Chou and G.-G. Chang, Reversible unfolding of the severe acute respiratory syndrome coronavirus main protease in guanidinium chloride, Biophys. J., 2007, 92(4), 1374-1383.

16 B. K. Park, A. Boobis, S. Clarke, C. E. P. Goldring, D. Jones, J. G. Kenna, C. Lambert, H. G. Laverty, D. J. Naisbitt, 
S. Nelson, et al., Managing the challenge of chemically reactive metabolites in drug development, Nat. Rev. Drug Discovery, 2011, 10(4), 292-306.

17 J. Degen, C. Wegscheid-Gerlach, A. Zaliani and M. Rarey, On the art of compiling and using'drug-like'chemical fragment spaces, ChemMedChem, 2008, 3(10), 1503-1507.

18 M. Su, Q. Yang, Y. Du, G. Feng, Z. Liu, Y. Li and R. Wang, Comparative assessment of scoring functions: The casf2016 update, J. Chem. Inf. Model., 2018.

19 L. Zhang, D. Lin, X. Sun, U. Curth, C. Drosten, L. Sauerhering, S. Becker, K. Rox and R. Hilgenfeld, Crystal structure of sars-cov-2 main protease provides a basis for design of improved $\alpha$-ketoamide inhibitors, Science, 2020, 368(6489), 409-412.

20 H. Su, S. Yao, W. Zhao, M. Li, L. Jia, W. Shang, H. Xie, C. Ke, M. Gao, K. Yu, et al., Discovery of baicalin and baicalein as novel, natural product inhibitors of sars-cov-2 $3 \mathrm{cl}$ protease in vitro, bioRxiv, 2020.

21 H. Wang, S. He, W. Deng, Y. Zhang, G. Li, J. Sun, W. Zhao, Y. Guo, Y. Zheng, D. Li, et al., Comprehensive insights into the catalytic mechanism of middle east respiratory syndrome $3 \mathrm{c}$-like protease and severe acute respiratory syndrome 3c-like protease, ACS Catal., 2020, 10(10), 58715890.

22 W. Dai, B. Zhang, X.-M. Jiang, H. Su, J. Li, Y. Zhao, X. Xie, Z. Jin, J. Peng, F. Liu, et al., Structure-based design of antiviral drug candidates targeting the sars-cov-2 main protease, Science, 2020, 368(6497), 1331-1335.

23 C. Ma, M. D. Sacco, B. Hurst, J. A. Townsend, Y. Hu, T. Szeto, X. Zhang, B. Tarbet, M. T. Marty, Y. Chen, et al., Boceprevir, gc-376, and calpain inhibitors ii, xii inhibit sars-cov-2 viral replication by targeting the viral main protease, bioRxiv, 2020.

24 A. Douangamath, D. Fearon, P. Gehrtz, T. Krojer, P. Lukacik, C. D. Owen, E. Resnick, C. Strain-Damerell, P. ÁbrányiBalogh, J. Brandaõ-Neto, et al., Crystallographic and electrophilic fragment screening of the sars-cov-2 main protease, Nat. Commun., 2020, 11, 5047.

25 U. Bacha, J. Barrila, S. B. Gabelli, Y. Kiso, L. M. Amzel and E. Freire, Development of broad-spectrum halomethyl ketone inhibitors against coronavirus main protease 3clpro, Chem. Biol. Drug Des., 2008, 72(1), 34-49.

26 A. K. Ghosh, M. Brindisi, D. Shahabi, M. E. Chapman and A. D. Mesecar, Drug development and medicinal chemistry efforts toward sars-coronavirus and covid-19 therapeutics, ChemMedChem, 2020, 15(11), 907-932.

27 P.-H. Liang, Characterization and inhibition of sarscoronavirus main protease, Curr. Top. Med. Chem., 2006, 6(4), 361-376.

28 H.-M. Wang and P.-H. Liang, Pharmacophores and biological activities of severe acute respiratory syndrome viral protease inhibitors, Expert Opin. Ther. Pat., 2007, 17(5), 533-546.

29 V. Kumar, Y.-S. Jung and P.-H. Liang, Anti-sars coronavirus agents: a patent review (2008-present), Expert Opin. Ther. Pat., 2013, 23(10), 1337-1348.
30 T. Pillaiyar, M. Manickam, V. Namasivayam, Y. Hayashi and S.-H. Jung, An overview of severe acute respiratory syndrome-coronavirus (sars-cov) $3 \mathrm{cl}$ protease inhibitors: peptidomimetics and small molecule chemotherapy, $J$. Med. Chem., 2016, 59(14), 6595-6628.

31 S. Ullrich and C. Nitsche, The sars-cov-2 main protease as drug target, Bioorg. Med. Chem. Lett., 2020, 127377.

32 Z. X. Cang, L. Mu and G. W. Wei, Representability of algebraic topology for biomolecules in machine learning based scoring and virtual screening, PLoS Comput. Biol., 2018, 14(1), e1005929, DOI: 10.1371/journal.pcbi.1005929.

33 D. Nguyen and G.-W. Wei, AGL-Score: Algebraic graph learning score for protein-ligand binding scoring, ranking, docking, and screening, J. Chem. Inf. Model., 2019, 59(7), 3291-3304.

34 G. Carlsson, Topology and data, Bull. Am. Math. Soc., 2009, 46(2), 255-308.

35 G. Jones, P. Willett, R. C. Glen, A. R. Leach and R. Taylor, Development and validation of a genetic algorithm for flexible docking, J. Mol. Biol., 1997, 267(3), 727-748.

36 O. Trott and A. J. Olson, AutoDock Vina: improving the speed and accuracy of docking with a new scoring function, efficient optimization, and multithreading, J. Comput. Chem., 2010, 31(2), 455-461.

37 R. A. Friesner, J. L. Banks, R. B. Murphy, T. A. Halgren, J. J. Klicic, D. T. Mainz, M. P. Repasky, E. H. Knoll, M. Shelley, J. K. Perry, et al., Glide: a new approach for rapid, accurate docking and scoring. 1. Method and assessment of docking accuracy, J. Med. Chem., 2004, 47(7), 1739-1749.

38 M. Wójcikowski, M. Kukiełka, M. Stepniewska-Dziubinska and P. Siedlecki, Development of a protein-ligand extended connectivity (plec) fingerprint and its application for binding affinity predictions, 2018.

39 D. D. Nguyen and G.-W. Wei, DG-GL: Differential geometrybased geometric learning of molecular datasets, Int. J. Numer. Method Biomed. Eng., 2019, 35(3), e3179.

40 L. Zheng, J. Fan and Y. Mu, Onionnet: a multiple-layer intermolecular-contact-based convolutional neural network for protein-ligand binding affinity prediction, ACS Omega, 2019, 4(14), 15956-15965.

41 H. Li, K.-H. Sze, G. Lu and P. J. Ballester, Machine-learning scoring functions for structure-based drug lead optimization, Wiley Interdiscip. Rev.: Comput. Mol. Sci., 2020, e1465.

42 D. D. Nguyen, Z. Cang and G.-W. Wei, A review of mathematical representations of biomolecular data, Phys. Chem. Chem. Phys., 2020, 22(8), 4343-4367.

43 J. Jiménez, M. Skalic, G. Martínez-Rosell and G. De Fabritiis, K DEEP: Protein-Ligand absolute binding affinity prediction via 3D-convolutional neural networks, J. Chem. Inf. Model., 2018, 58(2), 287-296.

44 K. Wu and G. W. Wei, Quantitative Toxicity Prediction Using Topology Based Multitask Deep Neural Networks, J. Chem. Inf. Model., 2018, 58, 520-531. 